\title{
Performance of Blind Equalization with Higher Order Statistics in Indoor Radio Environments
}

\author{
José Luis Valenzuela, Student, IEEE, Antonio Valdovinos, Student, IEEE, and Fernando J. Casadevall, Member, IEEE
}

\begin{abstract}
This paper analyzes the performance of blind equalization using the complex cepstrum of third-order moments applied to 4-QAM time division multiple access (TDMA) indoor radio communication systems. In particular, we have modeled a dispersive indoor channel with Rice statistics. We used the blind algorithms to estimate the channel-impulse response, and from this, we computed the equalizer coefficients using a classical minimum mean square error (MMSE) algorithm. In order to evaluate the system performance, we calculated the bit error rate (BER) of a decision feedback equalizer (DFE) that uses a tricepstrum algorithm to estimate the channel-impulse response. The results are compared with those obtained using a least sum of square errors (LSSE) algorithm as a channel estimator and considering the exact channel response. The results obtained show that this kind of blind equalizer performs better than the more classically trained equalizer when Rice channels with a strong direct path and signal-to-noise ratios (SNR's) lower than $20 \mathrm{~dB}$ are taken into account. However, some problems relating to the length of time needed for convergence must be solved.
\end{abstract}

Index Terms - Channel estimator, data communications, equalizers, multipath channel, multiple access.

\section{INTRODUCTION}

D IGITAL indoor radio communication systems have become an attractive proposal because they could provide the user with high-quality voice and medium-rate data services with total mobility. However, such systems are affected by frequency-selective fading caused by the multipath timedelay spread that produces intersymbol interference (ISI), thus resulting in an irreducible bit error rate (BER) and imposing an upper limit on the data symbol rate. Consequently, for time division multiple access (TDMA) systems, adaptive equalization has traditionally been adopted in order to counteract the degradation introduced by the channel. In general, most equalizers have two modes of operation: training and decision-directed [1]. In the training mode, the algorithm uses a known sequence to ensure that the equalizer coefficients achieve the appropriate values to reduce the ISI. However, the use of these training bits involves a major reduction of the payload efficiency; for this reason it is very interesting to investigate algorithms that allow equalization of the indoor channel without needing a training sequence. Even though there are several works that analyze the behavior of blind equalization [2]-[4], they deal with theoretical channels that do

Manuscript received March 4, 1995; revised December 1, 1995. This paper was supported by the CICYT, Spain, under Grant TIC 93-1079-CE.

The authors are with the Departament de Teoria del Senyal i Comunicacions, E.T.S. Ingenieros de Telecomunicación, Universitat Politécnica de Catalunya, 08080 Barcelona, Spain (e-mail: valens@hpgrc7.upc.es).

Publisher Item Identifier S 0018-9545(97)03117-4. not offer a realistic vision of a transmission system in indoor environments.

In this work, we used the properties of the complex cepstrum of higher order cumulants to obtain an accurate estimation of the impulse response of the channel. The subsequent calculation of the equalizer coefficients is done analytically. We simulated an indoor channel as a Rice statistics channel on the basis of measurements carried out in various environments [5]. We will assess the performance of 4-QAM (quadratureamplitude modulation) indoor radio systems with a decision feedback equalizer (DFE), whose coefficients are computed from the estimation of the impulse response by using the minimum mean square error (MMSE) criterion. We limited our study to the DFE structure because linear transversal equalizers cannot compensate frequency zeros that might appear in the transfer function of the mobile radio channels, resulting in performances that are worse than those obtained for DFE structures [1].

In order to evaluate the performance of the tricepstrum algorithm, we calculated the BER of the system as a function of the signal-to-noise ratio (SNR) and also of the delay spread that measures the channel dispersion. We also compared the difference in the performance of the blind system and a system that uses the least sum of square errors (LSSE) channel-estimation algorithm [6]. This algorithm is based on a correlation procedure with a training sequence and has been chosen for the universal mobile telecommunication system (UMTS) [7], [8]. Finally, we will evaluate the degradation introduced by the channel-impulse response estimation by computing the performance achieved by a system that uses the exact impulse response.

\section{TRANSMISSION MODEL}

Fig. 1 shows the low-pass equivalent model of the transmission system. In general, the transmitted $M$-QAM signal can be formulated as

$$
s(t)=\sum_{k=-\infty}^{\infty}\left(a_{k}+j b_{k}\right) \delta(t-k T)=\sum_{k=-\infty}^{\infty} c_{k} \delta(t-k T)
$$

where $\left\{a_{k}\right\}$ and $\left\{b_{k}\right\}$ are independent data sequences for the in-phase and quadrature channel. These data sequences take their values from the set $\pm 1, \pm 3, \cdots, \pm\left(M^{1 / 2}-1\right)$ with $M=4$ for 4 QAM. The overall filtering transfer function $H_{N}(f)=H_{T}(f) H_{R}(f)$ is a raised-cosine-type with a rolloff factor $\alpha$ equal to 0.5 , which is split equally between the transmitter and receiver. The function $H_{c}(f)$ models the 


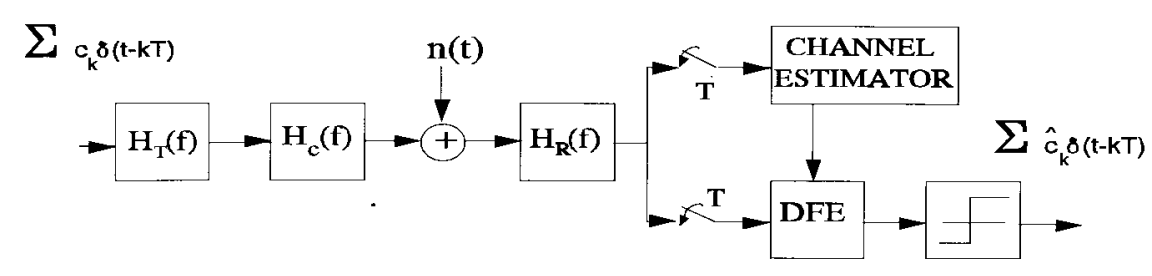

Fig. 1. Baseband model of the transmission system.

channel behavior that introduces selective fading in the radio link. The received signal can be expressed as

$$
r(t)=\sum_{k=-\infty}^{\infty} c_{k} h(t-k T)+n_{f}(t)
$$

where $n_{f}(t)$ is the Gaussian filtered noise added by the channel and $h(t)$ is the overall impulse response given by

$$
\begin{aligned}
h(t) & =h_{T}(t) * h_{c}(t) * h_{R}(t) \\
& =\mathcal{F}^{-1}\left\{H_{T}(f) H_{R}(f)\right\} * h_{c}(t) \\
& =\mathcal{F}^{-1}\left\{H_{N}(f)\right\} * h_{c}(t)
\end{aligned}
$$

where

$$
h_{c}(t)=\mathcal{F}^{-1}\left\{H_{c}(f)\right\}
$$

and

$$
\mathcal{F}^{-1}\left\{H_{N}(f)\right\}=h_{N}(t)=\frac{\sin \left(\frac{\pi t}{T}\right)}{\frac{\pi t}{T}} \frac{\cos \left(\frac{\beta \pi t}{T}\right)}{1-\left(\frac{2 \beta t}{T}\right)^{2}}
$$

$\mathcal{F}^{-1}$ being the inverse Fourier transform operator, $\beta$ the rolloff factor of the raised-cosine function, and $T$ the symbol period.

In this paper, we have considered a quasi-static Rice channel model. In order to generate the channel-impulse response, we can formulate this impulse response as

$$
h_{c}(t)=\alpha \delta(t)+h_{c c}(t)
$$

where $h_{c c}(t)$ models the dispersive part of a Rice channel represented by a Rayleigh stochastic process. We have assumed that the dispersive Rayleigh part of the model could be represented by means of a wide-sense stationary uncorrelated scattering (WSSUS) model, and it is represented by a unique correlation function referred to as the power-delay profile, $P(t)$, [1]. From the power-delay profile function, a sample of the dispersive part of the channel-impulse response can be constructed using the following formula [9]:

$$
h_{c c}(t)=\sqrt{T_{m}} \sum_{n=1}^{D}\left(h_{i n}+j h_{q n}\right) \delta\left(t-n T_{m}\right)
$$

where $h_{i n}$ and $h_{q n}$ are zero-mean Gaussian random variables with variance $P\left(n T_{m}\right) / 2$, where $T_{m}$ is the time between samples and $D$ is the number of samples needed to represent the indoor mobile channel in an accurate form. The value of $D$ depends on the shape of the power-delay profile, $P(t)$, and on its rms delay spread [1]. The most common shape for the power-delay profile in indoor environments is the oneside exponential profile. In order to compute the minimum value of $D$ in the channel model necessary to characterize the channel behavior, we should consider that the mean value of the received symbol energy must be equal to one, that is, we normalize the energy of the received signal. Then, computing from the channel model the mean of the symbol energy against the number of samples per symbol period and considering different values of the rms delay spread, we can conclude that the minimum sample period must be $T_{m}=\tau / 2$. Furthermore, it is sufficient to consider a time duration of a one-side exponential profile approximately equal to $14 \tau$, that is, $D$ is equal to 28 . To generate the Rice channel, we added a constant value to the first sample [10]. This constant value is represented in (6) by the delta function, whose amplitude $\alpha$ is a function of a magnitude $K$, defined as the ratio between the directed $\left(S_{d}\right)$ and reflected $\left(S_{r}\right)$ power of the channel-impulse response components. That is

$$
K=\frac{S_{d}}{S_{r}}=\frac{\alpha^{2}}{\sum_{n=0}^{D} P\left(n T_{m}\right)} \Rightarrow \alpha=\sqrt{K \sum_{n=0}^{D} P\left(n T_{m}\right)}
$$

Taking into account that the mean power of the received signal can be formulated as

$$
P_{m}=S_{d}+S_{r}=S_{d}\left(\frac{K+1}{K}\right)=\alpha^{2}\left(\frac{K+1}{K}\right)
$$

and considering that the $\mathrm{SNR}$ is given by $\mathrm{SNR}=P_{m} / \sigma_{n}^{2}$, where $\sigma_{n}^{2}$ is the noise power, then the noise-standard derivation used in the simulation procedure can be computed as

$$
\sigma_{n}=\sqrt{\frac{K+1}{\mathrm{SNR}}\left[\sum_{n=0}^{D} P\left(n T_{m}\right)\right]}
$$

Finally, the received and sampled signal at the input equalizer can be formulated as

$$
r\left(n T_{m}\right)=\sum_{k=-\infty}^{\infty} c_{k} h\left[(n-k) T_{m}\right]+n_{f}(k T)
$$

where $n_{f}\left(l T_{m}\right)$ is a complex Gaussian random uncorrelated process [9], with variance equal to $\sigma_{n}^{2}$, and $h\left[(l-k) T_{m}\right]$ is the sampled response of the channel model given by

$$
\begin{aligned}
h\left[(l-k) T_{m}\right] \equiv & \alpha h_{N}\left[(l-k) T_{m}\right] \\
& +\sum_{n=1}^{D}\left(h_{\subset}+j h_{q n}\right) h_{N}\left[(l-k) T_{m}\right]
\end{aligned}
$$


with

and

$$
h_{N}\left[(l-k) T_{m}\right]=\frac{\sin (\pi \gamma)}{\pi \gamma} \frac{\cos (\beta \pi \gamma)}{1-(2 \beta \gamma)^{2}}
$$

$$
\gamma=(l-k) \frac{T_{m}}{T}
$$

\section{High-ORder Statistics EQUALIZER}

The technique used to compensate the fast and selective fading effects of the mobile channel is based on the calculation of the equalizer coefficients using the MMSE algorithm from a channel-impulse estimation obtained with the tricepstrum algorithm.

The impulse-response estimation can be calculated by means of the algorithm that follows. First, we calculate the fourth-order cumulant given by

$$
R_{r}(m, n, l)=E\{r(k) r(k+m) r(k+n) r(k+l)\}
$$

where $r(k)$ is the sequence of the received signal samples. It is important to emphasize that the estimation of the fourthorder cumulant requires a long sequence in order to compute this function with sufficient accuracy to obtain the required precision on the impulse-response estimation. Next, using the properties of cumulants [11] and assuming that the channel can be modeled as

$$
H(z)=\prod_{i=1}^{L_{1}}\left(1-d_{i} z^{-1}\right) \prod_{i=1}^{L_{2}}\left(1-e_{i} z\right)=I(z) P(z)
$$

where we have divided the impulse response in a minimum and maximum phase impulse response with $\left|d_{i}\right|<1$ and $\left|e_{i}\right|<1$; the cepstral equation [11] can be reached

$$
\begin{aligned}
\sum_{I=1}^{P} & \left\{D^{(I)}\left[R_{r}(m-I, n, l)-R_{r}(m+I, n+I, l+I)\right]\right\} \\
& +\sum_{J=1}^{q}\left\{E ^ { ( J ) } \left[R_{r}(m-J, n-J, l-J)\right.\right. \\
& \left.\left.-R_{r}(m+J, n, l)\right]\right\}=-m R_{r}(m, n, l)
\end{aligned}
$$

In the above expression, $D^{(I)}$ and $E^{(I)}$ are the minimum and maximum phase-differential cepstrum coefficients, which are related to the zeros of the transfer function of $H(z)$ as follows:

$$
D^{(k)}=\sum_{i=1}^{L_{1}} d_{i}^{k} E^{(k)}=\sum_{i=1}^{L_{2}} e_{i}^{k}
$$

$d_{i}$ and $e_{i}$ being the coefficients of the $Z$ transform of impulse response $h(t)$ defined in (16). The values of $L_{1}$ and $L_{2}$ determine the number of estimated samples of the channel as $L_{1}+L_{2}+1$ [11] and therefore affect both the system performance and computational complexity.
Finally, the values of $D_{i}$ and $E_{i}$ are obtained from (17) by resolving a linear overdetermined system of equations, as shown in [2], and then, by means of a recursive procedure, the minimum and maximum phase impulse responses are computed as

$$
\begin{array}{r}
i(n)=-\frac{1}{n} \sum_{k=2}^{n+1} D^{(k-1)} i(n-k+1) \\
n=1,2,3, \cdots, M \\
p(n)=-\frac{1}{n} \sum_{k=2}^{n+1}-E^{(1-k)} p(n-k+1) \\
n=-1,-2,-3, \cdots, M
\end{array}
$$

where $i(0)=p(0)=1$. From these sets of values, the components of the global estimate response $\hat{h}(t)$ are obtained as follows:

$$
\hat{h}(n)=\sum_{k=0}^{M} i(k) p(n-k)=i(n) * p(n)
$$

where

$$
\hat{h}(t)=\sum_{n} \hat{h}_{r}(n)+j \hat{h}_{i}(n) \delta(t-n T)
$$

and $*$ is the convolution operator.

We used the MMSE criterion in order to obtain the coefficients from the impulse-response estimation because it performs better than the zero-forcing criterion in mobile communications environments. When quasi-static radio channels are assumed, a set of linear equations can be posed to obtain the optimum tap values [12], as shown in (23) at the bottom of the page, and

$$
C_{i}=-\sum_{l=-N}^{0} C_{l} h_{i-l} \quad i=1, \cdots, M
$$

where $\left\{C_{l}\right\}$ is the set of the equalizer tap, $h_{i}$ are the values of the impulse-response estimation at the sampling instant $t=i T, \sigma_{d}$ is the data-standard deviation, and $R_{N N}$ is the autocorrelation matrix of the noise given by

$$
R_{N N}(i-l)= \begin{cases}\sigma_{n}^{2}, & i=l \\ 0, & i \neq l\end{cases}
$$

\section{RESULTS}

We have examined the effectiveness of the counteracting tricepstrum adaptive equalization technique in the multipath indoor radio channel. The objective is to determine the datarate limitation for indoor communication systems. The criterion used to evaluate the system quality is the BER. In order to test the performance of the proposed algorithm in typical indoor environments, a simulation was performed. For every

$$
\sum_{l=-N}^{0} C_{l}\left[\sum_{n=-\infty}^{\infty} h_{n}^{*} h_{n+i-l}-\sum_{j=1}^{M} h_{i-l} h_{j-i}^{*}+\frac{1}{\sigma_{d}^{2}} R_{N N}(i-l)\right]=h_{-i}^{*} \quad i=-N, \cdots, 0
$$






Fig. 2. BER versus the normalized delay spread for a channel with low direct path.

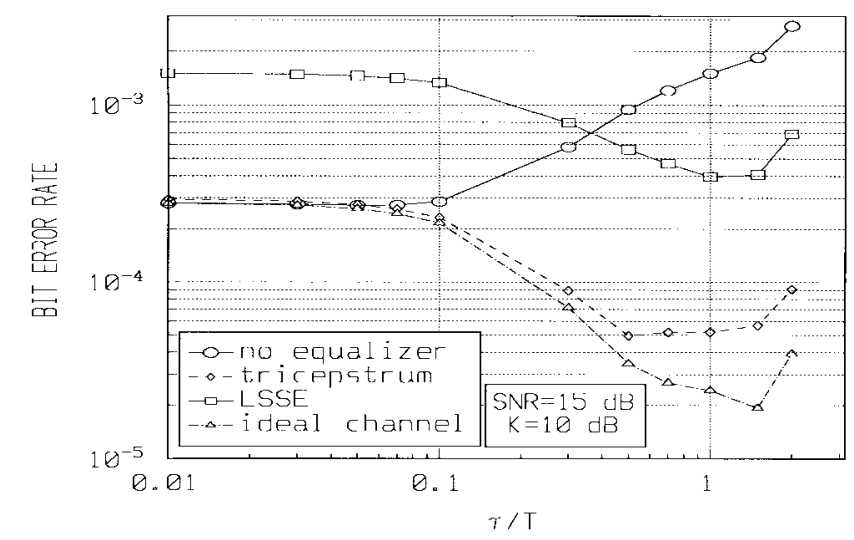

Fig. 3. BER versus the normalized delay spread for a channel with high direct path.

environment condition considered, which is specified by the SNR, the normalized delay spread, and the $K$ factor of the Rice channel model; a set of 1000 different impulse responses are generated, and the equalizer coefficients are calculated. Then, for each impulse response assumed, the equalizer tap values are calculated, the BER values are evaluated using the Levy method [13], and from these values, the mean value of the bit error probability is obtained for a given environment. Four different receiver structures were analyzed. First of all, we considered a system without an equalizer and compared the results obtained with the performance of a system with DFE's, where the estimated channel-impulse response is obtained from the tricepstrum channel estimation or from the LSSE channel-estimation algorithm, or, in the best case, from an ideal channel-estimation procedure. Specifically, 10000 received symbols per different channel environment were used to compute the tricepstrum algorithm, and from it, the equally time-sampled values of the channel-impulse response are estimated. We estimate nine taps of the sampled channel-impulse response for both the tricepstrum and LSSE because this is the value used in the UMTS approach [7].

In Figs. 2-7, we compare the results obtained with a DFE with five forward taps and four feedback taps and consider a 4-QAM modulation scheme.
Figs. 2 and 3 show the mean-value evolution of the bit error probability against the normalized delay-spread value $\tau / T$, where $T$ is the symbol period, for a Rice environment with two values of the directed-to-reflected power ratio $(K=0 \mathrm{~dB}$ and $K=10 \mathrm{~dB}$ ), and $15 \mathrm{~dB}$ of SNR. These figures show that for very low dispersive channels $(\tau / T<0.1)$, the tricepstrum algorithm has almost the same performance as a system with an equalizer, whose coefficients have been calculated from a perfect (ideal) estimation of the channel-impulse response. It can be noticed that when the dispersion grows, the tricepstrum algorithm does not reach the minimum obtained by the exact equalizer, and the system degradation increases mainly for channels with low power in the direct path, that is, with small values of the $K$ parameter.

If we compare the above results with those obtained using the LSSE algorithm, it is also clear that the higher order algorithm performs considerably better than the LSSE estimator for low dispersion channels and with a small directed path. It should be emphasized that for low values of the SNR, the LSSE algorithm causes major degradation of the BER performances, even for a very low dispersion channel, due to the noisy estimation of the sampled impulse response. The fact that the performances of a system with this kind of algorithm are even worse that those obtained for a system without an equalizer prevents us from using the LSSE algorithm in such an environment. However, in high dispersive channels, e.g., $\tau / T>0.5$ in Fig. 2, the tricepstrum performs worse than the LSSE procedure. This is due to the poles and zeros of the channel-transfer functions that are placed near the circle unit in the $Z$ plane, that is, values of $e_{i}$ and $d_{i}$ modules near one. However, when we have a high directed path $(K=10 \mathrm{~dB})$, as is usual in indoor environments, the tricepstrum algorithm works considerably better than the LSSE algorithm, with low losses in relation to the ideal situation, even for $\tau / T$ values as high as 0.5, as is shown in Fig. 3. Notice that, in this case, the tricepstrum algorithm produces BER values of one order of magnitude better than the more classical LSSE algorithm. It is also important to emphasize that for $K=10 \mathrm{~dB}$, the system performance for both receiver algorithms is better than that obtained for a lower direct-path power, $K=0 \mathrm{~dB}$, as could be expected, but the improvement obtained is greater when the tricepstrum algorithm is used.

The BER evolution versus SNR is depicted in Figs. 4 and 5 for the same values of $K$, where we can see that for an SNR lower than $15 \mathrm{~dB}$, the tricepstrum algorithm does not produce significant losses in comparison with the ideal system, whereas the LSSE algorithm produces a degradation on the required SNR value, for a given BER value, ranging from 2 to $4 \mathrm{~dB}$. However, for channels with a high direct path, $K=10$ for instance, and high SNR's (SNR > $20 \mathrm{~dB}$ ), the LSSE algorithm clearly outperforms the tricepstrum algorithm.

In Fig. 6, the BER evolution, as a function of the number of symbols used to train the equalizer, is shown for two channel environments. From these results, we can conclude that a large number of symbols, around 3000, is necessary to achieve BER values similar to the ones obtained with the LSSE algorithm. This is due to the need for a good estimation of the fourth-order cumulant. When the number of symbols is 


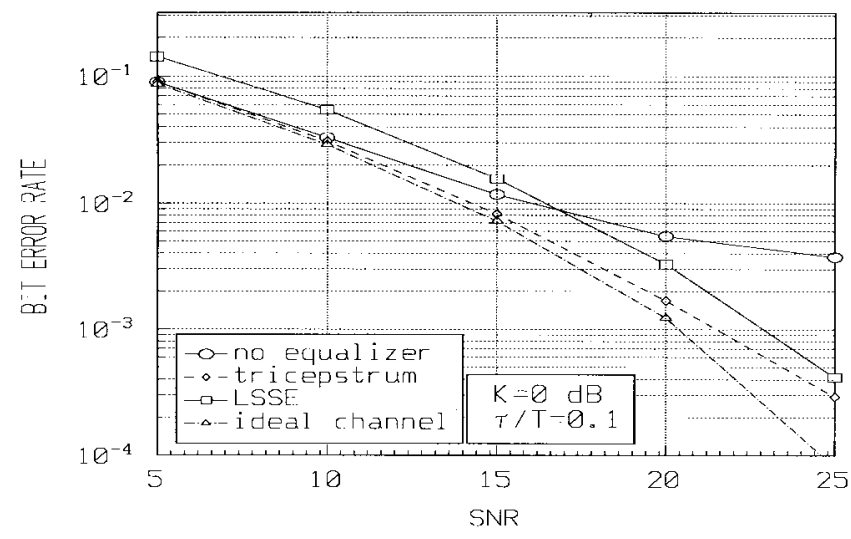

Fig. 4. BER versus the mean value of the SNR. Evolution for channels with low direct path.

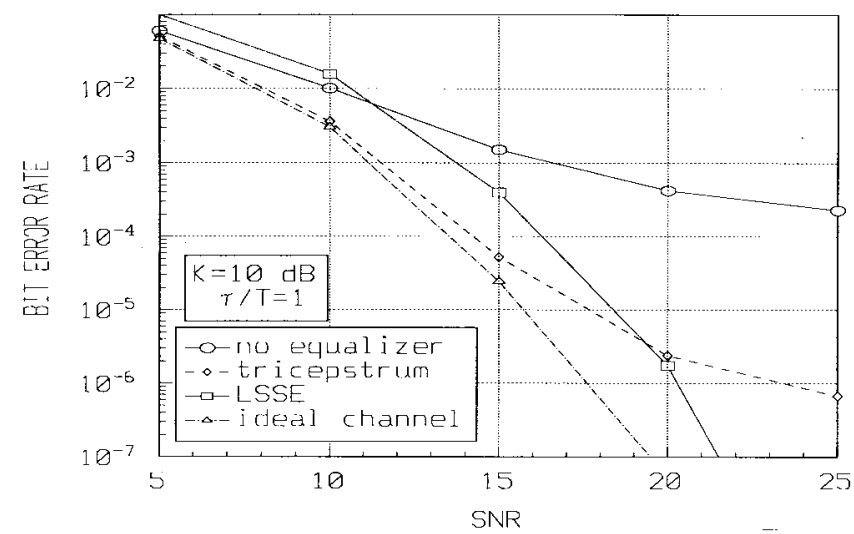

Fig. 5. BER versus the mean value of the SNR. Evolution for channel with high direct path.

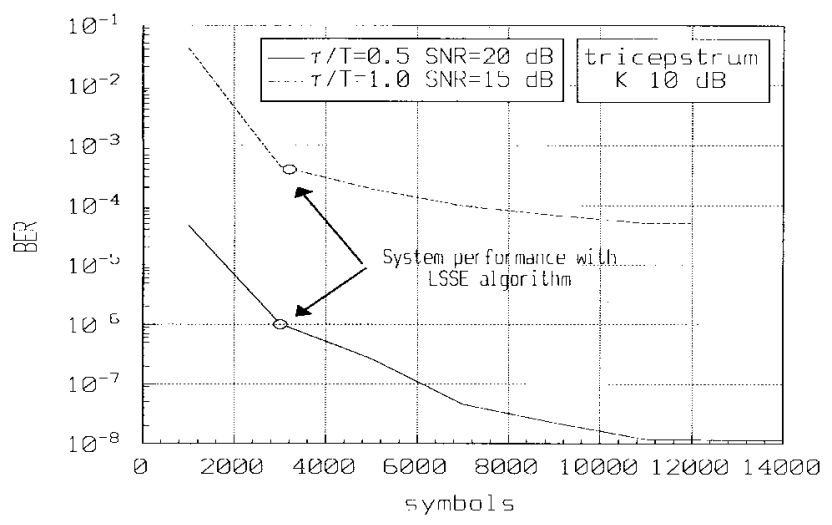

Fig. 6. Influence of the number of symbols used to compute the estimated impulse response on the accuracy to compute the mean value of the BER.

increased to 10000 or more, the BER falls to final values of around $10^{-8}$ for $\tau / T=0.5$ and $\mathrm{SNR}=20 \mathrm{~dB}$ and $5 \cdot 10^{-5}$ for $\tau=1.0$ and SNR $=15 \mathrm{~dB}$; values very near to those obtained when we compute the system performance with the ideally estimated impulse response (see Fig. 5). That is, this algorithm needs at least 8000 or 10000 symbols to achieve a good channel-impulse response estimation.

We also obtained results for the BER against the $K$ factor. In Fig. 7, we show the system performance for SNR $=10$

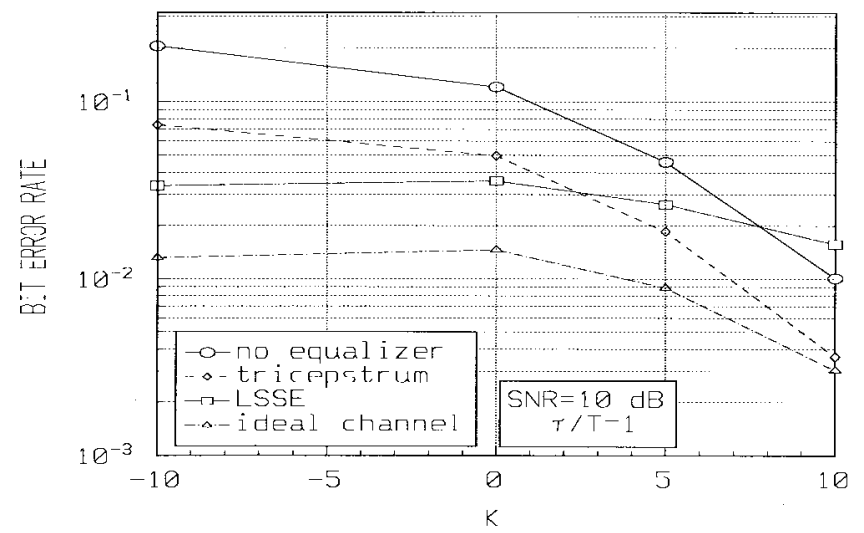

Fig. 7. Influence on the BER of direct path power ( $K$ value).

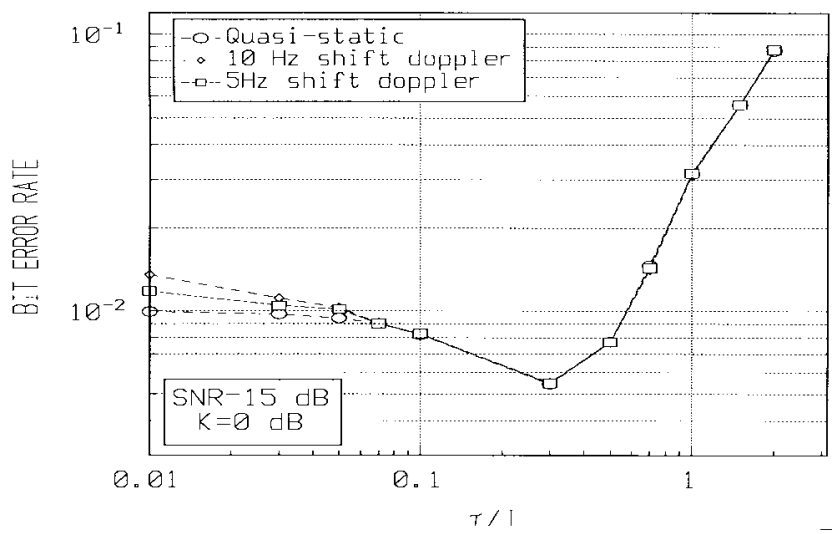

Fig. 8. Influence of the Doppler shift.

and $\tau / T=1$. We observe that LSSE works better when we do not have a direct path, that is, in Rayleigh environments, but the tricepstrum algorithm is more suitable when the channel has Rice statistics, even for values of $K$ as small as $3 \mathrm{~dB}$, performing near an ideal impulse-response channel estimator for high values of $K$.

It is important to notice that the above results were obtained with a quasi-static channel model. This approximation is valid in indoor environments, where we have a maximum Doppler shift of $10 \mathrm{~Hz}$ and at a high data rate. In Fig. 8, we show the differences in the results of the simulation of the indoor channel caused by considering or underestimating the Doppler effect. It can be seen that there is minimal deviation in the curves, and it is produced at a low rate when the effect of the equalizer is almost null.

\section{CONCLUSIONS}

In this paper, the performance of high-order statistical techniques in an indoor radio environment has been analyzed. It has been shown that the algorithm offers effectiveness for adaptive equalizing in the situation analyzed and provides a viable solution to the problem of channel distortion in indoor digital communication systems. This algorithm does not need a training sequence, but it does need a long data sequence to achieve a good estimation of the channel. Therefore, we 
can use this algorithm mainly for blind equalization in the downlink (base-to-mobile), where the receiver can use the continuous data frame in order to estimate and equalize the distorted channel, but usually not in the uplink (mobile-tobase), due to the discontinuous nature of the burst structure in most of the systems envisaged.

We have also analyzed the behavior of the algorithm with realistic channels and compared it with that of the LSSE estimator. From the results obtained, we can conclude that for low and medium dispersive channels, the tricepstrum algorithm works better than the LSSE. This algorithm has a good performance when the SNR is lower than $20 \mathrm{~dB}$ and when the channel has a strong direct path. However, with respect to the classical algorithms, there are several problems that must be solved, such as the long time convergence and the estimation of channels whose frequency response has zeros near the circle unit.

\section{REFERENCES}

[1] J. G. Proakis, Digital Communications, 2nd ed. New York: McGrawHill, 1982.

[2] D. Hatzinakos and C. L. Nikias, "Blind equalization using a tricepstrunbased algorithm," IEEE Trans. Commun., vol. 39, no. 5, pp. 669-682, 1991.

[3] A. Benveniste and M. Goursat, "Blind equalizers," IEEE Trans. Commun., vol. 32, pp. 871-883, Aug. 1994.

[4] D. Hatzinakos and C. L. Nikias, "Blind decision feedback equalization structures based on adaptive cumulant techniques," in Proc. ICC '89, Boston, MA, pp. 1278-1282.

[5] R. J. C. Bultitude, "Measurement, characterization and modeling of indoor 800/900 MHz radio channels for digital communications," IEEE Commun. Mag., vol. 25, no. 6, pp. 5-12, 1987.

[6] S. N. Crozier, D. D. Falconer, and S. A. Mahmoud, "Least sum of squared errors (LSSE) channel estimation," Proc. Inst. Elect. Eng., vol. 138, pt. F, no. 4, pp. 371-378, 1991.

[7] A. Urie, M. Streeton, and C. Mourot, "An advanced TDMA mobile access system for UMTS," in PIMRC '94, Hague, The Netherlands, pp. 685-690.

[8] J. Wolfman, "Almost perfect autocorrelation sequences," IEEE Trans. Inform. Theory, vol. 38, no. 4, pp. 1412-1418, 1992.

[9] M. Jeruchim, P. Balaban, and K. Shannmugan, Simulation of Communication Systems. New York: Plenum, 1992.

[10] K. Shannmugan and A. M. Brepohl, Random Signals. New York: Wiley, 1984.

[11] R. Pan and C. L. Nikias, "The complex cepstrum on higher order cumulants and nonminimum phase system identification," IEEE Trans. Acoust., Speech Signal Processing, vol. 36, pp. 186-205, Feb. 1988.

[12] A. P. Clark, Equalizers for Digital Modems. London: Pentech, pp. 205-217.

[13] A. Levy, "Fast error evaluation in the presence of intersymbol interference," IEEE Trans. Commun., vol. COMM-33, no. 5, pp. 479-481, 1985.



José Luis Valenzuela (S'93) was born in Teruel, Spain, in 1968. He received the Engineer of Telecommunications degree from the Escola Tècnica Superior d'Enginyers de Telecomunicació de Barcelona (ETSETB), Universitat Politècnica de Catalunya (UPC), Barcelona, Spain, in 1993. He is currently pursuing the $\mathrm{Ph} . \mathrm{D}$. degree in the area of communications systems at ETSETB.

In 1993, he joined ETSETB, where he has been an Associate Professor in the Signal Theory and Communications Department, UPC. His research interest is in the field of digital mobile radio communications systems.

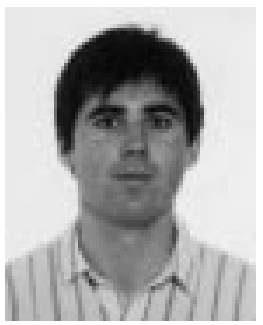

Antonio Valdovinos (S'91) was born in Barbastro, Spain, in 1966. He received the Engineer of Telecommunications and $\mathrm{Ph} . \mathrm{D}$. degrees from the Escola Tècnica Superior d'Enginyers de Telecomunicació de Barcelona (ETSETB), Universitat Politècnica de Catalunya (UPC), Barcelona, Spain, in 1990 and 1994, respectively.

In 1991, he joined the Signal Theory and Communications Department (UPC) under a research grant, where he was an Associate Professor until 1995. He is currently an Associate Professor at the Centro Politècnico Superior, Universidad de Zaragoza, Zaragoza, Spain. His research interest is in the field of digital mobile radio communications systems.

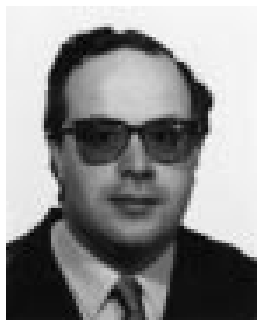

Fernando J. Casadevall ( $\left.M^{\prime} 87\right)$ was born in Barcelona, Spain, in 1955. He received the Engineer of Telecommunication and Ph.D. degrees from the Escola Tècnica Superior d'Enginyers de Telecomunicació de Barcelona (ETSETB), Universitat Politécnica de Catalunya (UPC), Barcelona, Spain, in 1977 and 1983, respectively.

In 1978, he joined ETSETB, where he was an Associate Professor from 1983 to 1991. He is currently a Full Professor in the Signal Theory and Communications Department, UPC. After graduation, he was concerned with equalization techniques for digita fiber optic systems. He has also been working in the field of digital communications with particular emphasis on digital radio and its performance under multipath propagation conditions. In the last ten years, he has mainly been concerned with the performance analysis and development of digital mobile radio systems. In particular, his research interests include cellular and personal communication systems, multipath receiver design, and digital signal processing. He has published about 40 papers and participated in about 20 funded projects, both private and government, on these fields. In particular, he has participated in the European research programs COST231 and RACE (ATDMA and CODIT projects), and he was also responsible for the Communications Technology Area in ANEP-CYCIT (Spanish Research Council) from October 1992 to January 1996. Currently, he is participating in the ACTS RAINBOW project as Leader of the UPC team. 